\title{
Singularity of generalized grey Brownian motions with different parameters
}

\author{
José Luís da Silva, \\ CIMA, University of Madeira, Campus da Penteada, \\ 9020-105 Funchal, Portugal. \\ Email: luis@uma.pt \\ Mohamed Erraoui \\ Université Cadi Ayyad, Faculté des Sciences Semlalia, \\ Département de Mathématiques, BP 2390, Marrakech, Maroc \\ Email: erraoui@uca.ma
}

November 13, 2018

\begin{abstract}
In this note we prove that the probability measures generated by two generalized grey Brownian motions with different parameters are singular with respect to each other. This result can be interpreted as an extension of the Feldman-Hájek dichotomy of Gaussian measures to a family of nonGaussian measures.
\end{abstract}

Keywords: Generalized grey Brownian motion, Fractional Brownian motion, $p$-variation, Mixing, Ergodicity.

\section{Introduction}

According to the Feldman-Hájek dichotomy (cf. Fel58, Háj58]) a pair of Gaussian measures on the space of functions on an interval are either mutually singular or equivalent. This result has led to numerous attempts to obtain convenient criteria for deciding between the two possibilities. In a paper on estimation of the Hurst index for fractional Brownian motion (fBm for short) Kur03, Kurchenko derived a Baxter-type theorem for the $\mathrm{fBm}$ based on the second order increments of the process. Based on Kurchenko's result, Prakasa Rao, in PR08, has investigated sufficient conditions under which probability measures generated by two fBms are singular with respect to each other. Inspired by the work of Cameron and Martin [CM47, we provide another proof of Prakasa Rao's result for fBm. To this end, we use the results on the variation of fBm from Rogers, cf. Rog97. This is the content of Section 2 . 
In the second part of this work we establish a similar result for a $(\alpha, \beta)$-family of non-Gaussian measures, so-called generalized grey noise measures (ggnm) $\mu_{\alpha, \beta}$ associated to the generalized grey Brownian motion $(\mathrm{ggBm}) B_{\alpha, \beta}$. To accomplished that, at first we study the $p$-variation of the ggBm and then prove the singularity of two ggnm with different parameters, cf. Section 3 .

\section{Singularity of fractional Brownian motions with different Hurst indices}

Let $\left\{W_{H}(t), t \in[0,1]\right\}$ be standard fBm with Hurst index $H \in(0,1)$ defined on a complete probability space $(\Omega, \mathcal{F}, P)$. Let $C([0,1]):=(C([0,1]), \mathcal{B})$ be the space of continuous functions with the $\sigma$-algebra $\mathcal{B}$ generated by the cylinder sets. We denote by $P_{W_{H}}$ the probability measure induced by $W_{H}$ on $C([0,1])$. The self-similarity of the $\mathrm{fBm}$ and the ergodic theorem imply that the $\mathrm{fBm}$ has $1 / H$-variation on the time interval $[0,1]$, see Rogers Rog97]. That is, with probability 1 , we have

$$
\lim _{n \rightarrow \infty} \sum_{j=1}^{2^{n}}\left|W_{H}\left(\frac{j}{2^{n}}\right)-W_{H}\left(\frac{j-1}{2^{n}}\right)\right|^{1 / H}=\mathbb{E}\left(\left|W_{H}(1)\right|^{1 / H}\right)=: \mu_{H} .
$$

Equivalently, for $P_{W_{H}}$ almost all $x \in C([0,1])$, we have

$$
\lim _{n \rightarrow \infty} \sum_{j=1}^{2^{n}}\left|x\left(\frac{j}{2^{n}}\right)-x\left(\frac{j-1}{2^{n}}\right)\right|^{1 / H}=\mu_{H} .
$$

For each $p \in] 0,+\infty)$ we introduce the following notations:

1. Let $D^{p}$ be the subset of $C([0,1])$ given by

$$
D^{p}:=\left\{x \in C([0,1])\left|\lim _{n \rightarrow \infty} \sum_{j=1}^{2^{n}}\right| x\left(\frac{j}{2^{n}}\right)-\left.x\left(\frac{j-1}{2^{n}}\right)\right|^{p} \text { does not exist }\right\} .
$$

2. For each $\lambda \geq 0$ let $C_{\lambda}^{p}$ be the subset of $C([0,1])$ defined by

$$
C_{\lambda}^{p}:=\left\{x \in C([0,1])\left|\lim _{n \rightarrow \infty} \sum_{j=1}^{2^{n}}\right| x\left(\frac{j}{2^{n}}\right)-\left.x\left(\frac{j-1}{2^{n}}\right)\right|^{p}=\lambda\right\} .
$$

Now we give some properties satisfied by the sets $C_{\lambda}^{p}, \lambda \geq 0$ and $D^{p}$.

(i) The sets $C_{\lambda}^{p}, \lambda \geq 0$ and $D^{p}$ are all disjoint, and

$$
C([0,1])=\left(\bigcup_{0 \leq \lambda} C_{\lambda}^{p}\right) \cup D^{p}
$$


(ii) For all $q>p$ and $\lambda \geq 0$, we have

$$
C_{\lambda}^{p} \subset C_{0}^{q} .
$$

Indeed, for any $x \in C_{\lambda}^{p}$ yields

$$
\begin{aligned}
\sum_{j=1}^{2^{n}}\left|x\left(\frac{j}{2^{n}}\right)-x\left(\frac{j-1}{2^{n}}\right)\right|^{q} & \leq\left(\sup _{1 \leq j \leq 2^{n}}\left|x\left(\frac{j}{2^{n}}\right)-x\left(\frac{j-1}{2^{n}}\right)\right|^{q-p}\right) \\
& \times \sum_{j=1}^{2^{n}}\left|x\left(\frac{j}{2^{n}}\right)-x\left(\frac{j-1}{2^{n}}\right)\right|^{p} .
\end{aligned}
$$

Now using the uniform continuity of $x$ in $[0,1]$, the right hand side of (5) converge to 0 as $n \rightarrow \infty$. Hence the inclusion (4) follows.

(iii) By passing at complements in (4), we equivalently have

$$
\left(\bigcup_{0<\lambda} C_{\lambda}^{q}\right) \cup D^{q} \subset D^{p}, \quad \forall q>p .
$$

An interpretation of (ii) and (iii) is: if the $p$-variation exists and it is nonzero then for any $q>p$ the $q$-variation is zero and for any $q<p$ the $q$-variation is infinite.

A a consequence of (2), the $P_{W_{H}}$-measures of the sets $C_{\lambda}^{1 / H}, \lambda \geq 0$ and $D^{1 / H}$ are as follows:

Lemma 1. The $P_{W_{H}}$-measures of the sets $C_{\lambda}^{1 / H}, \lambda \geq 0$ and $D^{1 / H}$ are:

$$
P_{W_{H}}\left(\bigcup_{0 \leq \lambda \neq \mu_{H}} C_{\lambda}^{1 / H}\right)=P_{W_{H}}\left(D^{1 / H}\right)=0, \quad P_{W_{H}}\left(C_{\mu_{H}^{1 / H}}^{1 / H}\right)=1 .
$$

Let $\left\{W_{H_{i}}(t), t \in[0,1]\right\}, i=1,2$ be two fBms with Hurst indices $H_{1} \neq H_{2}$. We will now show that the probability measures induced by these processes $P_{W_{H_{1}}}, P_{W_{H_{2}}}$ are singular with respect to each other. We state it in Theorem 3 below. The proof is based on the following lemma.

Lemma 2. Assume that $H_{1}<H_{2}$. Then we have

1. $C_{\mu_{H_{2}}}^{1 / H_{2}} \subset C_{0}^{1 / H_{1}}$,

2. $C_{\mu_{H_{1}}}^{1 / H_{1}} \subset D^{1 / H_{2}}$.

Proof. The assertions 1. and 2. are consequences of (ii) and (iii) respectively.

Now we are able to state the main result of this section

Theorem 3. The probability measures $P_{W_{H_{1}}}$ and $P_{W_{H_{2}}}$ are singular with respect to each other.

Proof. It follows from Lemmas 1 and 2 that $P_{W_{H_{1}}}\left(C_{\mu_{H_{2}}}^{1 / H_{2}}\right)=P_{W_{H_{2}}}\left(\left(C_{\mu_{H_{2}}}^{1 / H_{2}}\right)^{c}\right)=$ 0 , which means that the probability measures $P_{W_{H_{1}}}$ and $P_{W_{H_{2}}}$ are singular. 


\section{Generalized grey Brownian motion}

Let $0<\alpha<2$ and $0<\beta \leq 1$ be given. A continuous stochastic process is a generalized grey Brownian motion noted by $\left\{B_{\alpha, \beta}, t \geq 0\right\}$, if :

1. $B_{\alpha, \beta}(0)=0 P$-a.s.

2. Any collection $X=\left\{B_{\alpha, \beta}\left(t_{1}\right), \ldots, B_{\alpha, \beta}\left(t_{n}\right)\right\}$ with $0 \leq t_{1}<t_{2}<\ldots<$ $t_{n}<\infty$ has characteristic function given by

$$
\begin{aligned}
& \mathbb{E}\left(\exp \left(i \sum_{i=1}^{n} \theta_{i} B_{\alpha, \beta}\left(t_{i}\right)\right)\right)=E_{\beta}\left(-\frac{1}{2} \theta^{\top} \Sigma_{\alpha} \theta\right), \\
& \theta=\left(\theta_{1}, \ldots, \theta_{n}\right) \in \mathbb{R}^{n}, \Sigma_{\alpha}=\left(t_{i}^{\alpha}+t_{j}^{\alpha}-\left|t_{i}-t_{j}\right|^{\alpha}\right)_{i, j=1}^{n}
\end{aligned}
$$

and the joint probability density function is given by:

$$
f_{\alpha, \beta}\left(\theta, \Sigma_{\alpha}\right)=\frac{(2 \pi)^{-\frac{n}{2}}}{\sqrt{\operatorname{det} \Sigma_{\alpha}}} \int_{0}^{\infty} \tau^{-\frac{n}{2}} e^{-\frac{1}{2 \tau} \theta^{\top} \Sigma_{\alpha}^{-1} \theta} M_{\beta}(\tau) d \tau .
$$

Here $E_{\beta}$ is the Mittag-Leffler (entire) function

$$
E_{\beta}(z)=\sum_{n=0}^{\infty} \frac{z^{n}}{\Gamma(\beta n+1)}, \quad z \in \mathbb{C},
$$

and where $M_{\beta}$ is the so-called $M$-Wright probability density function with Laplace transform

$$
\int_{0}^{\infty} e^{-s \tau} M_{\beta}(\tau) d \tau=E_{\beta}(-s)
$$

The absolute moments of order $\delta>-1$ in $\mathbb{R}^{+}$are finite and turn out to be

$$
\int_{0}^{\infty} \tau^{\delta} M_{\beta}(\tau) d \tau=\frac{\Gamma(\delta+1)}{\Gamma(\beta \delta+1)}
$$

The generalized grey Brownian motion has the following properties:

1. For each $t \geq 0$, the moments of any order are given by

$$
\left\{\begin{array}{l}
\mathbb{E}\left(B_{\alpha, \beta}^{2 n+1}(t)\right)=0, \\
\mathbb{E}\left(B_{\alpha, \beta}^{2 n}(t)\right)=\frac{(2 n) !}{2^{n} \Gamma(\beta n+1)} t^{n \alpha} .
\end{array}\right.
$$

2. The covariance function has the form

$$
\mathbb{E}\left(B_{\alpha, \beta}(t) B_{\alpha, \beta}(s)\right)=\frac{1}{2 \Gamma(\beta+1)}\left(t^{\alpha}+s^{\alpha}-|t-s|^{\alpha}\right), \quad t, s \geq 0 .
$$


3. For each $t, s \geq 0$, the characteristic function of the increments is

$$
\mathbb{E}\left(e^{i \theta\left(B_{\alpha, \beta}(t)-B_{\alpha, \beta}(s)\right)}\right)=E_{\beta}\left(-\frac{\theta^{2}}{2}|t-s|^{\alpha}\right), \quad \theta \in \mathbb{R}
$$

All these properties may be summarized as follows. For any $0<\alpha<2$ and $0<\beta \leq 1$, the $\operatorname{ggBm} B_{\alpha, \beta}(t), t \geq 0$, is $\frac{\alpha}{2}$-self-similar with stationary increments. We refer to MM09 for the proof and more details. This class includes $\mathrm{fBm}$ for $\beta=1$, and Brownian motion $(\mathrm{Bm})$ for $\alpha=\beta=1$. We note also that Equation (6) shows that ggBm, which is not Gaussian in general, is a stochastic process defined only through its first and second moments which is a property of Gaussian processes.

Finally, it was shown in MP08 that the $\operatorname{ggBm} B_{\alpha, \beta}$ admits the following representation

$$
\left\{B_{\alpha, \beta}(t), t \geq 0\right\} \stackrel{d}{=}\left\{\sqrt{Y_{\beta}} B_{H}(t), t \geq 0\right\},
$$

where $\stackrel{d}{=}$ denotes the equality of the finite dimensional distribution and $B_{H}$ is a standard $\mathrm{fBm}$ with Hurst parameter $H=\alpha / 2 . \quad Y_{\beta}$ is an independent nonnegative random variable with probability density function $M_{\beta}(\tau), \tau \geq 0$.

\subsection{The p-variation of generalized grey Brownian motion}

This subsection is devoted to the study of the $p$-variation of ggBm. The approach taken is inspired from the one used for the fBm. To do this we will need to establish some properties satisfied by the $\operatorname{ggBm} B_{\alpha, \beta}$.

Lemma 4. The stationary sequence $\left(B_{\alpha, \beta}(j)-B_{\alpha, \beta}(j-1)\right)_{j \geq 1}$ is mixing. Therefore it is ergodic.

Proof. Since the process $B_{\alpha, \beta}(t), t \geq 0$, has stationary increments then the sequence $\left(B_{\alpha, \beta}(j)-B_{\alpha, \beta}(j-1)\right)_{j \geq 1}$ is stationary. To show that it is also mixing, it is sufficient to prove the decay of correlations

$$
\lim _{j \rightarrow+\infty} \operatorname{Cov}\left(f\left(B_{\alpha, \beta}(1)\right), g\left(B_{\alpha, \beta}(j)-B_{\alpha, \beta}(j-1)\right)\right)=0,
$$

for all bounded measurable $f, g$. It follows from the representation (11) that

$$
\begin{aligned}
\operatorname{Cov} & \left(f\left(B_{\alpha, \beta}(1)\right), g\left(B_{\alpha, \beta}(j)-B_{\alpha, \beta}(j-1)\right)\right) \\
& =\int_{0}^{\infty} M_{\beta}(\tau) \operatorname{Cov}\left(f\left(\tau^{1 / 2} B_{H}(1)\right), g\left(\tau^{1 / 2}\left(B_{H}(j)-B_{H}(j-1)\right)\right)\right) d \tau .
\end{aligned}
$$

It is well known that the sequence $\left(B_{H}(j)-B_{H}(j-1)\right)_{j>1}$ is stationary, centered Gaussian with covariance function satisfying

$$
\operatorname{Cov}\left(B_{H}(1)-B_{H}(0), B_{H}(j)-B_{H}(j-1)\right) \underset{j \rightarrow+\infty}{\longrightarrow} 0 .
$$


Therefore it is mixing, that is

$$
\lim _{j \rightarrow+\infty} \operatorname{Cov}\left(f\left(B_{H}(1)\right), g\left(\left(B_{H}(j)-B_{H}(j-1)\right)\right)\right)=0,
$$

for all bounded measurable $f, g$. Now (12) follows from the dominated convergence theorem.

Lemma 5. The sequence $\left(\frac{1}{n} \sum_{j=1}^{n}\left|B_{\alpha, \beta}(j)-B_{\alpha, \beta}(j-1)\right|^{p}\right)_{n \geq 1}$ converges a.s. (and in $L^{1}$ ) to $\mathbb{E}\left(\left|B_{\alpha, \beta}(1)\right|^{p}\right)$.

Proof. Since $\left(B_{\alpha, \beta}(j)-B_{\alpha, \beta}(j-1)\right)_{j \geq 1}$ is ergodic then as a consequence of the ergodic theorem (Theorem 3.3 p. 413 of [Shi96]), we obtain that $\left(\frac{1}{n} \sum_{j=1}^{n} \mid B_{\alpha, \beta}(j)-\right.$ $\left.\left.B_{\alpha, \beta}(j-1)\right|^{p}\right)_{n \geq 1}$ converge a.s. (and in $\left.L^{1}\right)$ to $\mathbb{E}\left(\left|B_{\alpha, \beta}(1)\right|^{p}\right)$.

As a consequence we obtain

Proposition 6. We have the following limit in probability

$$
\lim _{n \rightarrow+\infty} n^{p \frac{\alpha}{2}-1} \sum_{j=1}^{n}\left|B_{\alpha, \beta}\left(\frac{j}{n}\right)-B_{\alpha, \beta}\left(\frac{j-1}{n}\right)\right|^{p}=\mathbb{E}\left(\left|B_{\alpha, \beta}(1)\right|^{p}\right) .
$$

Proof. It follows from the $\frac{\alpha}{2}$-self-similarity of $B_{\alpha, \beta}(t), t \geq 0$, that for all $n \in \mathbb{N}$, we have the following equality in law

$Z_{n, p}:=n^{p \frac{\alpha}{2}-1} \sum_{j=1}^{n}\left|B_{\alpha, \beta}\left(\frac{j}{n}\right)-B_{\alpha, \beta}\left(\frac{j-1}{n}\right)\right|^{p}=\frac{1}{n} \sum_{j=1}^{n}\left|B_{\alpha, \beta}(j)-B_{\alpha, \beta}(j-1)\right|^{p}$.

This, together with the convergence in Lemma5 5 gives that $Z_{n, p}$ converges in law to $\mathbb{E}\left(\left|B_{\alpha, \beta}(1)\right|^{p}\right)$. Since the limit is a constant so the convergence in probability follows.

As a consequence of (ii) and (iii) we get the following result on the $p$-variation of the $\mathrm{ggBm}$.

Proposition 7. We have the following limit in probability

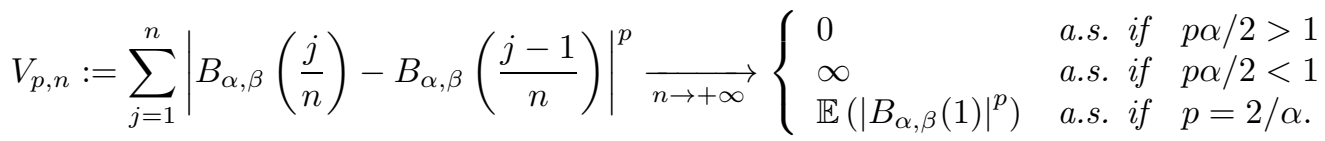

Remark 8. The ggBm is not a semimartingale. In addition, $B_{\alpha, \beta}$ cannot be of finite variation on $[0,1]$ and by scaling and stationarity of the increment on any interval. 
Proof. Indeed there is a subsequence such that $V_{p, n}$ converge almost surely to $\infty$ for $p=1$ and $\alpha \in(0,2)$. If $\alpha \in(1,2)$ we can choose $p \in(2 / \alpha, 2)$ such that $V_{p, n}$ converge to 0 for some subsequence. This implies that the quadratic variation of $B_{\alpha, \beta}$ is zero. If $\alpha \in(0,1)$ we can choose $p>2$ such that $2 p / \alpha<1$ and the $p$-variation of $B_{\alpha, \beta}$ must be infinite. So, in any case $B_{\alpha, \beta}$ can not be a semimartingale.

Remark 9. It follows from the representation (11) that the Hölder continuity of the trajectories of $\mathrm{ggBm}$ reduces to the Hölder continuity of the fBm. Thus, with probability 1 , we have

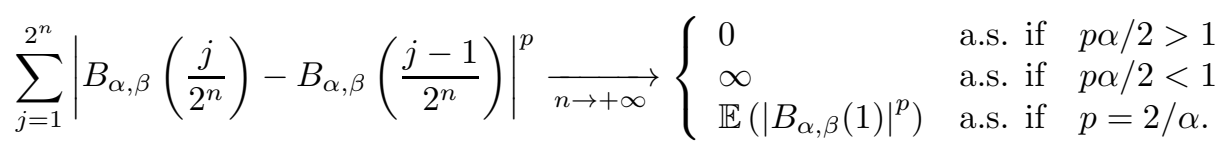

\subsection{Singularity of generalized grey Brownian motions with different parameters}

Since the law of the generalized grey Brownian motion is not Gaussian, FeldmanHájek dichotomy is no longer applicable. However, using the same approach as for the $\mathrm{fBm}$, we establish that the laws of two ggBm processes are singular if the parameters are different. Let us denotes by $P_{\alpha, \beta}$ (resp. $\left.P_{\alpha^{\prime}, \beta^{\prime}}\right)$ the probability measures generated by $B_{\alpha, \beta}$ (resp. $\left.B_{\alpha^{\prime}, \beta^{\prime}}\right)$ with $\alpha, \alpha^{\prime} \in(0,2)$ and $\beta, \beta^{\prime} \in(0,1]$.

Theorem 10. 1. For any $\beta, \beta^{\prime} \in(0,1]$ such that $\Gamma(\beta / \alpha+1) \neq \Gamma\left(\beta^{\prime} / \alpha+1\right)$, the probability measures $P_{\alpha, \beta}$ and $P_{\alpha, \beta^{\prime}}$ are singular with respect to each other for all $\alpha \in(0,2)$.

2. For $\alpha \neq \alpha^{\prime}$ then for any $\beta, \beta^{\prime} \in(0,1]$ the probability measures $P_{\alpha, \beta}$ and $P_{\alpha^{\prime}, \beta^{\prime}}$ are singular with respect to each other.

Remark 11. Before proceeding to the proof of the theorem we will give some examples of $\beta, \beta^{\prime} \in(0,1]$ for which the condition $\Gamma(\beta / \alpha+1) \neq \Gamma\left(\beta^{\prime} / \alpha+1\right)$ is verified. It is well known that there exists $\kappa \in(1,2)$ such that the $\Gamma$ function is strictly decreasing on $(0, \kappa]$ and strictly increasing on $[\kappa, \infty)$. So, for $\beta, \beta^{\prime} \in$ $(0, \alpha(\kappa-1) \wedge 1]$ or $\beta, \beta^{\prime} \in[\alpha(\kappa-1) \wedge 1,1]$ the condition is satisfied.

Proof of Theorem [10. It follows from Remark 9 that

$$
\lim _{n \rightarrow+\infty} \sum_{j=1}^{2^{n}}\left|x\left(\frac{j}{2^{n}}\right)-x\left(\frac{j-1}{2^{n}}\right)\right|^{2 / \alpha}=\mathbb{E}\left(\left|B_{\alpha, \beta}(1)\right|^{2 / \alpha}\right)=: \mu_{\alpha, \beta}, \quad P_{\alpha, \beta}-a . s .
$$

and

$$
\lim _{n \rightarrow+\infty} \sum_{j=1}^{2^{n}}\left|x\left(\frac{j}{2^{n}}\right)-x\left(\frac{j-1}{2^{n}}\right)\right|^{2 / \alpha^{\prime}}=\mathbb{E}\left(\left|B_{\alpha^{\prime}, \beta^{\prime}}(1)\right|^{2 / \alpha^{\prime}}\right)=: \mu_{\alpha^{\prime}, \beta^{\prime}}, P_{\alpha^{\prime}, \beta^{\prime}}-\text { a.s. }
$$

In other words, $P_{\alpha, \beta}\left(C_{\mu_{\alpha, \beta}}^{2 / \alpha}\right)=1$ and $P_{\alpha^{\prime}, \beta^{\prime}}\left(C_{\mu_{\alpha^{\prime}, \beta^{\prime}}}^{2 / \alpha^{\prime}}\right)=1$. 
1. For $(\alpha, \beta) \neq\left(\alpha, \beta^{\prime}\right)$, using the representation (11), the independence of $Y_{\beta}$ and $B_{H}$ and the moments formula (8) we obtain

$$
\mathbb{E}\left(\left|B_{\alpha, \beta}(1)\right|^{2 / \alpha}\right)=\frac{\Gamma(1 / \alpha+1)}{\Gamma(\beta / \alpha+1)} \mathbb{E}\left(\left|B_{H}(1)\right|^{2 / \alpha}\right),
$$

and

$$
\mathbb{E}\left(\left|B_{\alpha, \beta^{\prime}}(1)\right|^{2 / \alpha}\right)=\frac{\Gamma(1 / \alpha+1)}{\Gamma\left(\beta^{\prime} / \alpha+1\right)} \mathbb{E}\left(\left|B_{H}(1)\right|^{2 / \alpha}\right) .
$$

Since $\Gamma(\beta / \alpha+1) \neq \Gamma\left(\beta^{\prime} / \alpha+1\right)$ it is clear that for the limits (13) and (14) are different, that is $\mu_{\alpha, \beta} \neq \mu_{\alpha, \beta^{\prime}}$. The singularity of $P_{\alpha, \beta}$ and $P_{\alpha, \beta^{\prime}}$ follows from the fact that the sets $C_{\mu_{\alpha, \beta}}^{2 / \alpha}$ and $C_{\mu_{\alpha, \beta^{\prime}}}^{2 / \alpha}$ are disjoints.

2. For $\left(\alpha^{\prime}, \beta^{\prime}\right) \neq(\alpha, \beta)$ ( $\alpha<\alpha^{\prime}$ for example) it follows from (ii) that $C_{\mu_{\alpha^{\prime}, \beta^{\prime}}}^{2 / \alpha^{\prime}} \subset C_{0}^{2 / \alpha}$. Hence we deduce, from (i) that the measures $P_{\alpha, \beta}$ and $P_{\alpha^{\prime}, \beta^{\prime}}$ are singular with respect to each other.

\section{Acknowledgments}

We would like to thank the financial support of the Laboratory LIBMA form the University Cadi Ayyad Marrakech and the project I\&D: UID/MAT/04674/2013.

\section{References}

[CM47] R. H. Cameron and W. T. Martin. The behavior of measure and measurability under change of scale in Wiener space. Bull. Amer. Math. Soc., 53(2):130-137, 1947.

[Fel58] J. Feldman. Equivalence and perpendicularity of Gaussian processes. Pacific J. Math., 8(4):699-708, 1958.

[Háj58] J Hájek. On a property of normal distribution of any stochastic process. Czechoslovak Math. J., 8 (83):610-618, 1958.

[Kur03] O. O. Kurchenko. A consistent estimate of the Hurst parameter for a fractional Brownian motion. Theory Probab. Math. Statist., 67:97-106, 2003.

[MM09] A. Mura and F. Mainardi. A class of self-similar stochastic processes with stationary increments to model anomalous diffusion in physics. Integr. Transf. Spec. F., 20(3-4):185-198, 2009.

[MP08] A. Mura and G. Pagnini. Characterizations and simulations of a class of stochastic processes to model anomalous diffusion. J. Phys. A: Math. Theor., 41(28):285003, 22, 2008.

[PR08] B. L. S. Prakasa Rao. Singularity of fractional Brownian motions with different Hurst indices. Stoch. Anal. Appl., 26(2):334-337, January 2008 . 
[Rog97] L. C. G. Rogers. Arbitrage with fractional Brownian motion. Math. Finance, 7(1):95-105, January 1997.

[Shi96] A. N. Shiryaev. Probability. Springer Verlag, New York Berlin Heidelgerg, 1996. 